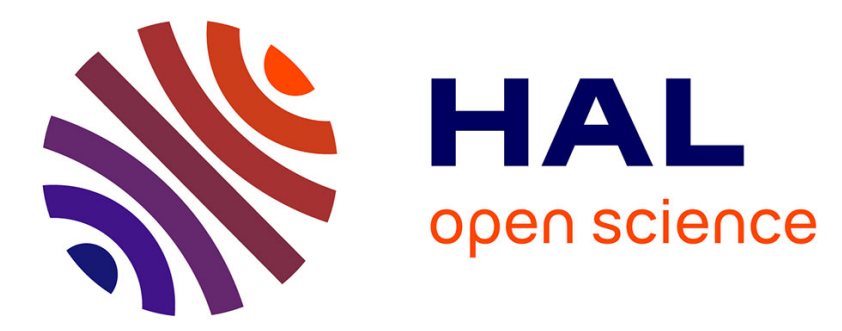

\title{
A robust circle criterion observer with application to neural mass models
}

Michelle Chong, Romain Postoyan, Dragan Nesic, Levin Kuhlmann, Andrea Varsavsky

\section{- To cite this version:}

Michelle Chong, Romain Postoyan, Dragan Nesic, Levin Kuhlmann, Andrea Varsavsky. A robust circle criterion observer with application to neural mass models. Automatica, 2012, 48 (11), pp.2986-2989. 10.1016/j.automatica.2012.08.008 . hal-00751770

\section{HAL Id: hal-00751770 \\ https://hal.science/hal-00751770}

Submitted on 23 Dec 2012

HAL is a multi-disciplinary open access archive for the deposit and dissemination of scientific research documents, whether they are published or not. The documents may come from teaching and research institutions in France or abroad, or from public or private research centers.
L'archive ouverte pluridisciplinaire HAL, est destinée au dépôt et à la diffusion de documents scientifiques de niveau recherche, publiés ou non, émanant des établissements d'enseignement et de recherche français ou étrangers, des laboratoires publics ou privés. 


\title{
A robust circle criterion observer with application to neural mass models. *
}

\author{
Michelle Chong $^{\text {a }}$, Romain Postoyan ${ }^{\mathrm{b}}$, Dragan Nešić ${ }^{\mathrm{a}}$, Levin Kuhlmann ${ }^{\mathrm{a}}$, \\ Andrea Varsavsky ${ }^{\mathrm{a}}$. \\ ${ }^{a}$ Department of Electrical and Electronic Engineering, The University of Melbourne, Australia. \\ ${ }^{\mathrm{b}}$ Centre de Recherche en Automatique de Nancy, UMR 7039, Nancy-Université, CNRS, France.
}

\begin{abstract}
A robust circle criterion observer is designed and applied to neural mass models. At present, no existing circle criterion observers apply to the considered models, i.e. the required linear matrix inequality is infeasible. Therefore, we generalise available results to derive a suitable estimation algorithm. Additionally, the design also takes into account input uncertainty and measurement noise. We show how to apply the observer to estimate the mean membrane potential of neuronal populations of a popular single cortical column model from the literature.
\end{abstract}

Key words: system state observer; linear matrix inequality approach; brain models; biomedical.

\section{Introduction}

The observation of states plays a significant role in many biological applications, most notably in brain research where the sensors that can be physically implanted cannot measure directly all variables of interest. The estimation of states is therefore especially useful for the diagnosis and classification of neurological diseases, as well as general neuroscientific studies for better understanding of the human brain [9].

We focus on models that describe the activity of neurons at the macroscopic level, i.e. the activity of populations of neurons. They are known in the literature as 'neural mass models' [3]. We consider a class of models that includes a model that describes the visual pathway when the brain is in an idle state [6], a model which replicates alpha rhythms [10] and a model that describes epileptic activity in the hippocampus [11]. The models mentioned originate from the seminal work of Lopes da Silva in [8]

\footnotetext{
* This paper was not presented at any IFAC meeting. Email addresses: chongms@unimelb.edu.au (Michelle Chong), romain.postoyan@cran.uhp-nancy.fr (Romain Postoyan), dnesic@unimelb.edu.au (Dragan Nešić), levink@unimelb.edu.au (Levin Kuhlmann), ava@unimelb.edu.au (Andrea Varsavsky).
}

and they all share the same mathematical structure:

$$
\begin{aligned}
& \dot{x}=A x+G \gamma(H x)+B u \\
& y=C x+D w
\end{aligned}
$$

where the state vector is $x \in \mathbb{R}^{n}$, the input is $u \in \mathbb{R}^{r}$, the measurement is $y \in \mathbb{R}^{p}$, the measurement noise is $w \in \mathbb{R}^{s}, A \in \mathbb{R}^{n \times n}, B \in \mathbb{R}^{n \times r}, C \in \mathbb{R}^{p \times n}, G \in \mathbb{R}^{n \times m}$, $H \in \mathbb{R}^{q \times n}, D \in \mathbb{R}^{p \times s}$ and $\gamma=\left(\gamma_{1}, \ldots, \gamma_{m}\right): \mathbb{R}^{q} \rightarrow \mathbb{R}^{m}$.

For the class of neural mass models considered, existing results in the literature for circle criterion observers [1], [5], [12] resulted in an infeasible linear matrix inequality (LMI) condition, which does not allow us to guarantee the convergence of the estimation error to the origin. Hence, we propose a generalised result that leads to feasible LMIs such that the observer can be applied to the models considered.

We also address two main issues faced in neuroscientific studies. Firstly, the input is not always measurable. Secondly, the measurements obtained are corrupted by noise. Hence, we improve the observer design in [2] by taking into account these two implementation issues. The resulting design allows observer gain matrices $L$ and $K$ to be obtained under the circle criterion, while taking the attenuation of input uncertainty and measurement noise into account. Our design differs from [12] in that 
we consider input uncertainty and we also introduce a multiplier $M$ in the LMI, so that the resulting observer is applicable to the class of neural mass models we consider.

Notation A vector $\left[\begin{array}{ll}a^{T} & b^{T}\end{array}\right]^{T}$ is denoted $(a, b)$, for all $a \in \mathbb{R}^{n_{a}}, b \in \mathbb{R}^{n_{b}}$. A block diagonal matrix with square matrices $A_{i} \in \mathbb{R}^{n_{i} \times n_{i}}$ is denoted as $\operatorname{diag}\left(A_{1}, \ldots, A_{n}\right)$. The identity matrix is denoted by $\mathbb{I}$. The symmetric block component of a symmetric matrix is denoted by $\star$. The vector norm of $f$ at each time $t$ is denoted $|f(t)|$. The $\mathcal{L}_{2}$ norm is defined as $\|f(t)\|_{2}=\left(\int_{0}^{t}|f(s)|^{2} \mathrm{ds}\right)^{\frac{1}{2}}$.

\section{A neural mass model}

As mentioned in the introduction, our results apply to a class of neural mass models. Due to space constraints, we choose to focus on a popular model found in [6]. This single cortical column model is able to generate realistic patterns such as alpha rhythms in the electroencephalogram (EEG), which we take as a measurement. It can be written in the form of (1) with the state vector $x=\left(x_{1}, \ldots, x_{8}\right)$. The variables $x_{j}, j \in\{1,3,5,7\}$ are the mean membrane potentials of the neuronal populations and $x_{k}, k \in\{2,4,6,8\}$ are their derivatives. The input $u \in \mathbb{R}$ is the afferent influence from other populations and is assumed in [6] to be a uniformly distributed signal between 120 and $320 \mathrm{mV}$. The output $y \in \mathbb{R}$ is the EEG measurement provided to the observer. All values of the constants in this section are non-negative and their physiological meaning can be found in [6]. The model is of the form (1) with:

$A=\operatorname{diag}\left(A_{1}, \ldots, A_{4}\right)$ where $A_{i}=\left[\begin{array}{cc}0 & 1 \\ -k_{i}^{2} & -2 k_{i}\end{array}\right]$,

$k_{1}=k_{3}=k_{4}=a$ and $k_{2}=b$, where $a, b>0$, $B=\left(0, \theta_{A} a, 0,0,0,0,0,0\right), C=\left[\begin{array}{llllllll}1 & 0 & -1 & 0 & 0 & 0 & 0 & 0\end{array}\right]$, $D=1, G=\left[\begin{array}{cccccccc}0 & \theta_{A} a C_{2} & 0 & 0 & 0 & 0 & 0 & 0 \\ 0 & 0 & 0 & \theta_{B} b C_{4} & 0 & 0 & 0 & 0 \\ 0 & 0 & 0 & 0 & 0 & \theta_{A} a C_{3} & 0 & \theta_{A} a C_{1}\end{array}\right]^{T}$, $H=\left[\begin{array}{cccccccc}0 & 0 & 0 & 0 & 0 & 0 & 1 & 0 \\ 0 & 0 & 0 & 0 & 1 & 0 & 0 & 0 \\ 1 & 0 & -1 & 0 & 0 & 0 & 0 & 0\end{array}\right], \gamma=(S, S, S)$. The sigmoid function is $S(s)=\frac{\alpha}{1+\exp \left(-r\left(s-V_{0}\right)\right)}$ for all $s \in \mathbb{R}$, where $\alpha, r>0$.

\section{Problem formulation}

In this section, we first state an assumption on system (1) which is satisfied by the considered neural mass models. We then set out our main objective. Suppose the nonlinearity $\gamma$ in (1) is both globally Lipschitz and monotonically increasing as follows:
Assumption 1 For any $i \in\{1, \ldots, m\}$, there exists constants $0 \leq a_{i} \leq b_{i}<\infty$, so that the following holds:

$$
a_{i} \leq \frac{\gamma_{i}\left(z_{i}\right)-\gamma_{i}\left(v_{i}\right)}{z_{i}-v_{i}} \leq b_{i}, \forall v_{i}, z_{i} \in \mathbb{R} \text { with } v_{i} \neq z_{i} .
$$

Assumption 1 is an extension of the slope restriction condition from [1, Equation (1)] to vector nonlinearity $\gamma=\left(\gamma_{1}, \ldots, \gamma_{m}\right)$. Constant $b_{i}$ is the Lipschitz constant of $\gamma_{i}$. We note that the function $\gamma$ specified in Section 2 satisfies Assumption 1 with $a_{1}=0$ and $b_{1}=\rho$, where $\rho=\frac{1}{2} \alpha r$ from the sigmoid function in Section 2 .

Note that from Assumption 1, we know that for any $i \in$ $\{1, \ldots, m\}$, there exists a time-varying gain $\delta_{i}(t)$ taking values in the interval $\left[0, b_{i}\right]$ (as done in $[1$, Equation (6)]) so that:

$$
\gamma_{i}\left(z_{i}\right)-\gamma_{i}\left(v_{i}\right)=\delta_{i}(t)\left(z_{i}-v_{i}\right), \forall v_{i}, z_{i} \in \mathbb{R} .
$$

We consider the following type of observer [1]:

$$
\dot{\hat{x}}=A \hat{x}+G \gamma(H \hat{x}+K(C \hat{x}-y))+L(C \hat{x}-y)+B(u+d) \text {, }
$$

where $\hat{x}$ is the state estimate, $d \in \mathbb{R}^{r}$ is the input disturbance and $K \in \mathbb{R}^{m \times p}, L \in \mathbb{R}^{n \times p}$ are the observer matrices to be designed.

As done in [1], denoting the observation error as $e:=$ $\hat{x}-x, v:=H x$ and $z:=H \hat{x}+K(C \hat{x}-y)$, the observation error system from (1) and (3) is $\dot{e}=(A+L C) e-L D w+$ $B d+G(\gamma(z)-\gamma(v))$. By (2), we obtain the observation error system as

$\dot{e}=(A+L C) e-L D w+B d+G \delta(t) \eta$,

where $\delta(t)=\operatorname{diag}\left(\delta_{1}(t), \ldots, \delta_{m}(t)\right)$ and $\eta:=z-v$.

Given the observation error system (4), our task is to find observer matrices $K$ and $L$ such that a quadratic Lyapunov function $V(e)$ satisfies the following along the solutions of (4):

$$
\dot{V}(e) \leq-|e|^{2}+\mu_{w}|w|^{2}+\mu_{d}|d|^{2} .
$$

We can then show that the observation error $e$ satisfies the following property ${ }^{1}$ for all $t \geq 0$ :

$$
\|e(t)\|_{2} \leq \bar{c}|e(0)|+\sqrt{\mu_{w}}\|w(t)\|_{2}+\sqrt{\mu_{d}}\|d(t)\|_{2},
$$

where scalars $\bar{c}, \mu_{w}, \mu_{d}>0$. The disturbance gains from $w$ and $d$ to $e$ are $\sqrt{\mu_{w}}$ and $\sqrt{\mu_{d}}$ respectively.

\section{A robust circle criterion observer}

In this section, we present the main result of this note. In Theorem 2, we establish that the observation error system (4) satisfies property (6) provided that a linear matrix inequality (LMI) is satisfied.

\footnotetext{
${ }^{1}$ We can obtain (6) from (5) by following the same procedure as in the proof of Theorem 5.2 in [7].
} 
Theorem 2 Consider system (1) and observer (3). Under Assumption 1, if there exist a real symmetric and positive definite matrix $P$, a diagonal and positive definite matrix $M=\operatorname{diag}\left(m_{1}, \ldots, m_{m}\right)$, and scalar constants $\mu_{w}, \mu_{d}>0$, such that the following is satisfied:

$$
\left[\begin{array}{cccc}
\mathcal{A}(P, P L) & \mathcal{B}\left(P, M, K^{T} M\right) & -P L D & P B \\
\star & \mathcal{E}(M) & -M K D & 0 \\
\star & \star & -\mu_{w} \mathbb{I} & 0 \\
\star & \star & \star & -\mu_{d} \mathbb{I}
\end{array}\right] \leq 0,
$$

where $\mathcal{A}(P, P L)=P(A+L C)+(A+L C)^{T} P+\mathbb{I}$, $\mathcal{B}\left(P, M, K^{T} M\right)=P G+(H+K C)^{T} M$ and $\mathcal{E}(M)=$ $-2 M \operatorname{diag}\left(\frac{1}{b_{1}}, \ldots, \frac{1}{b_{m}}\right)$, then the observation error system satisfies property (6).

The proof of Theorem 2 is provided in the Appendix. Theorem 2 shows that if a $K$ and $L$ can be found such that the LMI (7) is satisfied, then an observer (3) can be designed for system (1). Note that condition (7) is considered an LMI in $P, P L, M K, M, \mu_{w}$ and $\mu_{d}$. As such, (7) can be solved using efficient software tools such as the LMI Lab in MATLAB.

By considering the system (1) under the ideal condition where there is no input uncertainty and measurement error, we obtain the condition stated in Corollary 3 and obtained in [2, Theorem 2].

Corollary 3 Consider system (1) and observer (3) with $d=0$ and $w=0$. The origin of the observation error system (4) is globally exponentially stable if the following holds:

$$
\left[\begin{array}{cc}
\mathcal{A}(P, P L) & \mathcal{B}\left(P, M, K^{T} M\right) \\
\star & \mathcal{E}(M)
\end{array}\right] \leq 0,
$$

where $\mathcal{A}, \mathcal{B}$ and $\mathcal{E}$ are defined in Theorem 2.

Current circle criterion results in [1], [5], [12] yield LMIs that are not feasible for the class of neural models we consider. Therefore, we adapted [5] to the case where the nonlinearity $\gamma$ is globally Lipschitz and also monotonically increasing with inspiration from [1]. This result is a special case of the system considered in Theorem 2 as stated in Corollary 3 and was reported in [2]. In this note, we further improve the circle criterion observer obtained in [2] by designing observer matrices $K$ and $L$ under the circle criterion condition and taking input uncertainty $d$ from (3) and measurement noise $w$ from (1) into account. The LMI (7) differs from (13) obtained in [12] in the sense that we consider input uncertainty attenuation and introduced a multiplier $M$ in components $(1,2),(2,2)$ and $(2,3)$ of $(7)$. Without introducing the multiplier $M$, the results obtained in [12] do not lead to feasible LMIs. This simple extension allows circle criterion observers to be designed for the neural models we consider, in addition to taking into account the realistic issues faced when implementing these observers in the context of estimation for neuroscientific studies.

The constants $\mu_{w}$ and $\mu_{d}$ in (7) may be specified by the user and should the LMI (7) be found to be solvable, we then have the estimation error satisfying property (6) with estimates of the disturbance gains $\sqrt{\mu_{w}}$ and $\sqrt{\mu_{d}}$. In some cases, we may wish to minimise these constants and various methods are available to solve this multiobjective optimisation problem (see [4]). A simple approach that we take in the next section is to minimise the cost $J_{\max }=\max \left\{\mu_{w}, \mu_{d}\right\}$ subject to (7).

\section{Application to a neural mass model}

We introduce input disturbance $d \sim \mathcal{N}\left(0,0.1^{2}\right)$ and measurement noise $w \sim \mathcal{N}\left(0,0.7^{2}\right)$. The performance of (A) the circle criterion observer obtained under the conditions of Corollary 3 that does not consider the attenuation of input uncertainty and measurement noise is compared with (B) the robust circle criterion observer derived in Theorem 2. We solved LMI (8) to obtain observer matrices $K, L$ for observer (A). For observer (B), we choose to minimise $\mu_{w}$ and $\mu_{d}$ using the cost function $J_{\max }$ subject to $(7)$ to obtain $K$ and $L$. The resulting computed disturbance gains are $\sqrt{\mu_{w}}=706$ and $\sqrt{\mu_{d}}=9.48$. In the simulation that follows, we initialise the model at $x(0)=(6,0.5,6,0.5,6,0.5,6,0.5)$ and the observers at $\hat{x}(0)=0$. Figure 1 shows that the robust circle criterion observer obtained in Theorem 2 (Observer (B)) outperforms the observer obtained in Corollary 3 (Observer (A)) in the presence of input uncertainty and measurement noise.

\section{Conclusion}

We have designed a robust circle criterion observer that attenuates input uncertainty and measurement noise. The designed observer is then applied to a neural mass model that describes the generation of alpha rhythms prevalent in the cerebral cortex [6]. To the best of our knowledge, no other results in the literature leads to feasible LMIs.

\section{References}

[1] M. Arcak and P. Kokotović. Observer-based control of systems with slope-restricted nonlinearities. IEEE Transactions on Automatic Control, 46:1, 2001.

[2] M.S. Chong, R. Postoyan, D. Nešić, L. Kuhlmann, and A. Varsavsky. A circle criterion observer for estimating the unmeasured membrane potential of neuronal populations. In Proceedings of the Australian Control Conference, 2011. 

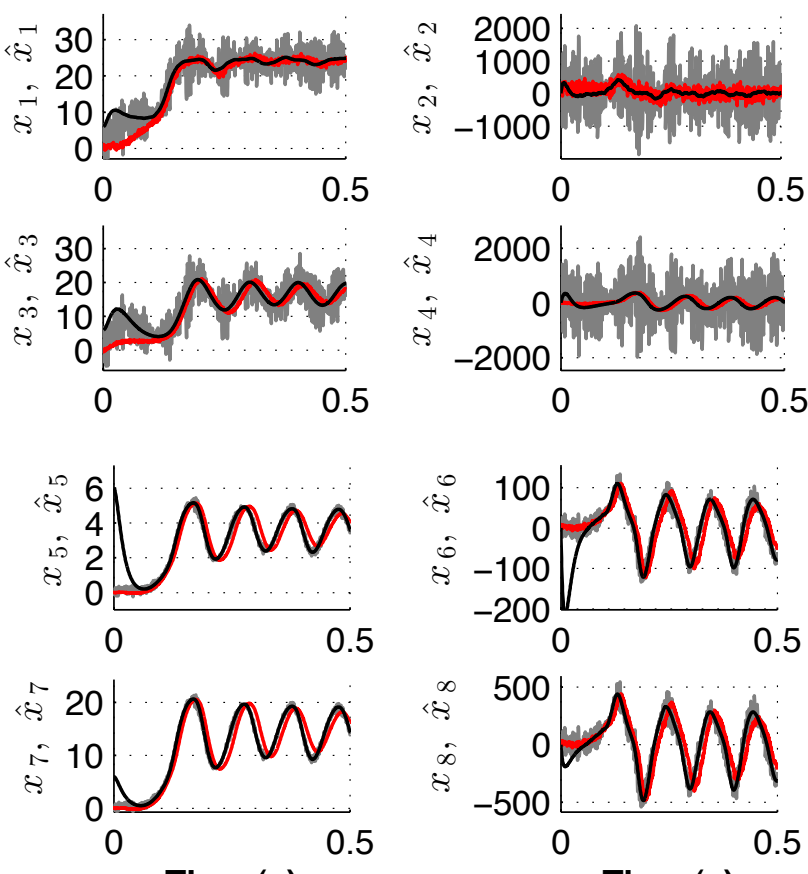

Time (s)

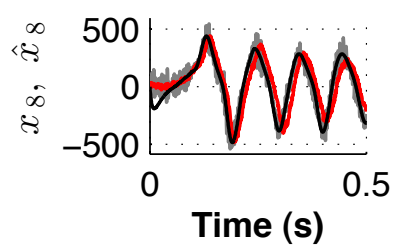

Fig. 1. Estimated states $\hat{x}$ converge to a neighbourhood of the true states $x$. Legend: Observer A (grey), Observer B (red) and Model (black).

[3] G. Deco, V. Jirsa, P.A. Robinson, M. Breakspear, and K. Friston. The dynamic brain: From spiking neurons to neural masses and cortical fields. Cerebral Cortex, 4(8):1-35, 2008.

[4] L. El Ghaoui and S.I. Niculescu. Advances in linear matrix inequality methods in control, volume 2. Society for Industrial Mathematics, 2000.

[5] X. Fan and M. Arcak. Observer design for systems with multivariable monotone nonlinearities. Systems \& Control Letters, 50(4):319 - 330, 2003.

[6] B.H. Jansen and V.G. Rit. Electroencephalogram and visual evoked potential generation in a mathematical model of coupled cortical columns. Biological Cybernetics, 73:357-366, 1995.

[7] H.K. Khalil. Nonlinear systems. Prentice Hall, 3rd edition, 2000 .

[8] FH Lopes da Silva, A. Hoeks, H. Smits, and LH Zetterberg. Model of brain rhythmic activity. Biological Cybernetics, 15(1):27-37, 1974

[9] S.J. Schiff. Neural Control Engineering: The Emerging Intersection Between Control Theory and Neuroscience. Computational Neuroscience. The MIT Press, 2011.

[10] C.J. Stam, J.P.M. Pijn, P. Suffczynski, and F.H. Lopes da Silva. Dynamics of the human alpha rhythm: evidence for non-linearity? Clinical Neurophysiology, 110(10):1801-1813, 1999.

[11] F. Wendling, A. Hernandez, J.J. Bellanger, P. Chauvel, and F. Bartolomei. Interictal to ictal transition in human temporal lobe epilepsy: insights from a computational model of intracerebral EEG. Journal of Clinical Neurophysiology, $22(5): 343,2005$.

[12] A. Zemouche and M. Boutayeb. A unified $H_{\infty}$ adaptive observer synthesis method for a class of systems with both
Lipschitz and monotone nonlinearities. Systems \& Control Letters, 58(4):282-288, 2009.

\section{A Proof of Theorem 2}

Firstly, $x(t)$ exists for all $t \geq 0$ by Theorem 3.2 of [7], because $\gamma$ is globally Lipschitz and $u$ is a continuous function that is defined for all $t \geq 0$. We now show that the observation error system satisfies property (6) by taking the derivative of the Lyapunov function $V(e)=e^{T} P e$ along the solutions of (4), where $\chi=(e, \delta(t) \eta, w, d)$ :

$$
\begin{aligned}
\dot{V}(e)= & e^{T}\left(P(A+L C)+(A+L C)^{T} P\right) e+2 e^{T} P G \delta(t) \eta \\
& -2 e^{T} P L D w+2 e^{T} P B d \\
= & \chi^{T}\left[\begin{array}{cccc}
P(A+L C)+(A+L C)^{T} P & P G & -P L D & P B \\
\star & 0 & 0 & 0 \\
\star & \star & 0 & 0 \\
\star & \star & \star & 0
\end{array}\right] \chi .
\end{aligned}
$$

Applying (7), we obtain:

$$
\begin{aligned}
\dot{V}(e) \leq \chi^{T}\left[\begin{array}{cccc}
-\mathbb{I}-(H+K C)^{T} M & 0 & 0 \\
\star & -\mathcal{E}(M) & M K D & 0 \\
\star & \star & \mu_{w} \mathbb{I} & 0 \\
\star & \star & \star & \mu_{d} \mathbb{I}
\end{array}\right] \chi \\
=-|e|^{2}-2 e^{T}(H+K C)^{T} M \delta(t) \eta+2 \eta^{T} \delta(t) M K D w \\
-\eta^{T} \delta(t)^{T} \mathcal{E}(M) \delta(t) \eta+\mu_{w}|w|^{2}+\mu_{d}|d|^{2} .
\end{aligned}
$$

Recall that $\eta:=z-v=(H+K C) e-K D w$, hence $(H+K C) e=\eta+K D w$. Therefore,

$$
\begin{aligned}
\dot{V}(e) \leq & -|e|^{2}-2(\eta+K D w)^{T} M \delta(t) \eta+2 \eta^{T} \delta(t) M K D w \\
& -\eta^{T} \delta(t)^{T} \mathcal{E}(M) \delta(t) \eta+\mu_{w}|w|^{2}+\mu_{d}|d|^{2} .
\end{aligned}
$$

Noting that $\delta(t)=\operatorname{diag}\left(\delta_{1}(t), \ldots, \delta_{n}(t)\right)=\delta(t)^{T}$,

$$
\begin{aligned}
\dot{V}(e) & +|e|^{2}-\mu_{w}|w|^{2}-\mu_{d}|d|^{2} \\
& \leq-2 \eta^{T}\left(M \delta(t)-\delta(t) M \operatorname{diag}\left(\frac{1}{b_{1}}, \ldots, \frac{1}{b_{m}}\right) \delta(t)\right) \eta .
\end{aligned}
$$

We examine $M \delta(t)-\delta(t) M \operatorname{diag}\left(\frac{1}{b_{1}}, \ldots, \frac{1}{b_{m}}\right) \delta(t)$ component by component, i.e. $\delta_{i}(t) m_{i}-\delta_{i}(t)^{2} m_{i} b_{i}{ }^{-1}=$ $\delta_{i}(t) m_{i}\left(1-\delta_{i}(t) b_{i}{ }^{-1}\right)$. As $\delta_{i}(t), m_{i}>0$ and by Assumption $1,1-\delta_{i}(t) b_{i}{ }^{-1} \geq 0$, we obtain $\delta(t) M-$ $\delta(t) M \operatorname{diag}\left(\frac{1}{b_{1}}, \ldots, \frac{1}{b_{m}}\right) \delta(t) \geq 0$. Hence, $\dot{V}(e)+|e|^{2}-$ $\mu_{w}|w|^{2}-\mu_{d}|d|^{2} \leq 0$. As explained in Section 3, this implies that the observation error system satisfies properties (6) as required. 